\title{
3. Artikel und Verweisstichwörter
}

Hermetica (K.-W. Tröger) 749

Katechumenat/Katechumenen (G. Kretschmar/K. Hauschildt).............

Kategorischer Imperativ $\rightarrow$ Kant, $\rightarrow$ Pflicht

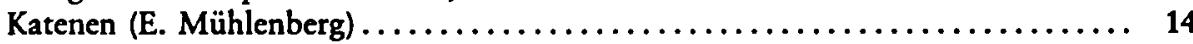

Katharer (D. Müller) ................................... 21

Katharina von Siena (G. Alberigo) $\ldots \ldots \ldots \ldots \ldots \ldots \ldots \ldots \ldots \ldots \ldots \ldots \ldots \ldots, 30$

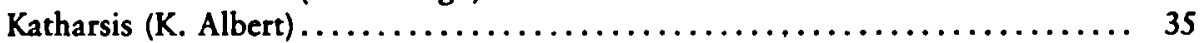

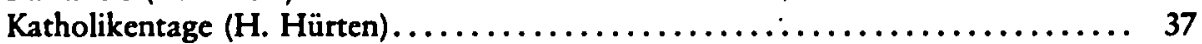

Katholisch-apostolische Gemeinde (H.-D. Reimer) $\ldots \ldots \ldots \ldots \ldots \ldots \ldots \ldots \ldots, 40$

Katholische Aktion (A. Steinmaus-Pollak) .................... 43

Katholische Briefe $\rightarrow$ Jakobusbrief, $\rightarrow$ Johannesbriefe, $\rightarrow$ Judasbrief, $\rightarrow$ Petrusbriefe

Katholische Reform und Gegenreformation (G. Maron).............. 45

Katholizismus $\rightarrow$ Römisch-katholische Kirche

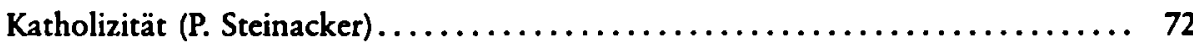

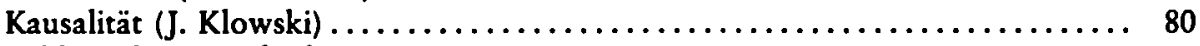

Keble, John $\rightarrow$ Oxford

Keduscha $\rightarrow$ Trishagion

Kelch $\rightarrow$ Abendmahl, $\rightarrow$ Geräte, Liturgische

Keltische Kirchen (R. Sharpe) $\ldots \ldots \ldots \ldots \ldots \ldots \ldots \ldots \ldots \ldots \ldots \ldots \ldots \ldots \ldots \ldots$

Keltische Religion (G. Lanczkowski) . ........................ 92

Keniter/Keniterhypothese $\rightarrow$ Nomadentum im Alten Testament, $\rightarrow$ Religionsgeschichte Israels

Kenose $\rightarrow$ Jesus Christus

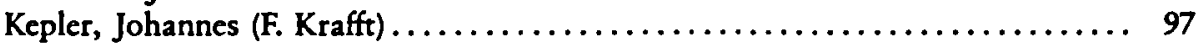

Kerygma $\rightarrow$ Bultmann, Rudolf, $\rightarrow$ Rechtfertigung, $\rightarrow$ Wort Gottes

Ketteler, Wilhelm Emmanuel von (E. Iserloh) . .................... 109

Ketzer/Ketzerbekämpfung $\rightarrow$ Häresie, $\rightarrow$ Inquisition

Ketzertaufstreit $\rightarrow$ Afrika, $\rightarrow$ Cyprian von Karthago, $\rightarrow$ Stephan I., $\rightarrow$ Taufe

Keuschheit (P. Gerlitz/W. Pratscher/P. F. Beatrice/J. Dominian) ............ 113

Kiel, Universität (H.-J. Birkner) . ........................... 134

Kierkegaard, Søren Aabye (H. Schröer) ......................... 138

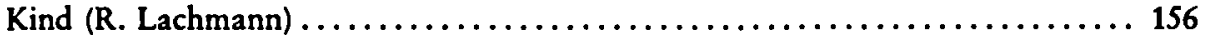

Kinderbibel (Ch. Reents) .................................. 176

Kinderfragen $\rightarrow$ Jugend, $\rightarrow$ Katechismus, $\rightarrow$ Kind

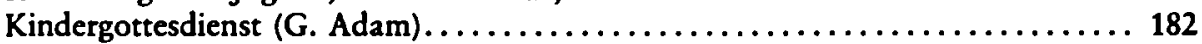

Kinderkommunion (E. Kenntner) $\ldots \ldots \ldots \ldots \ldots \ldots \ldots \ldots \ldots \ldots \ldots \ldots \ldots \ldots \ldots$

Kindertaufe $\rightarrow$ Taufe

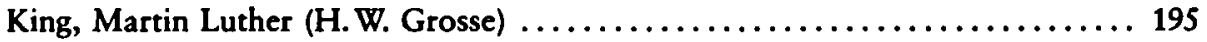

Kingo, Thomas Hansen $\rightarrow$ Gesangbuch, $\rightarrow$ Kirchenlied

Kingsley, Charles $\rightarrow$ Sozialismus

Kirche (K. Berger/G. May/J. Finkenzeller/A. Kallis/U. Kühn/W. Härle/

M. Honecker $/$ H. Schröer) .............................. 198

Kirche von England (O. Chadwick).......................... 344

Kirche und Staat (R.M. Grant/H.D. Betz/P. Moraw/V. Press/H. Kerner/

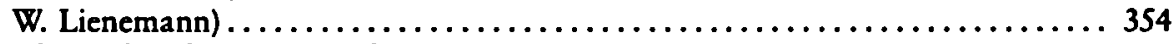

Kirche und Welt (M. Honecker) $\ldots \ldots \ldots \ldots \ldots \ldots \ldots \ldots \ldots \ldots \ldots \ldots \ldots \ldots, \ldots \ldots \ldots$

Kirchenaustritt $\rightarrow$ Kirchenentfremdung/Kirchenaustritte

Kirchenbau (H. Brandenburg/W. Haas/H. Hammer-Schenk/H. Schwebel) . . . . . 421

Kirchenbücher (H. Baier) ............................ 528

Kirchenbund $\rightarrow$ Kirchenverfassung

Kirchenentfremdung/Kirchenaustritte (A. Feige) $\ldots \ldots \ldots \ldots \ldots \ldots \ldots \ldots \ldots 530$ 
Kirchengeschichtsschreibung (E. Stöve) $\ldots \ldots \ldots \ldots \ldots \ldots \ldots \ldots \ldots \ldots \ldots, 535$

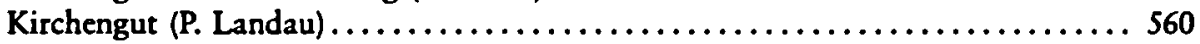

Kirchenhoheit $\rightarrow$ Kirchenregiment, Landesherrliches, $\rightarrow$ Kirchenverfassungen

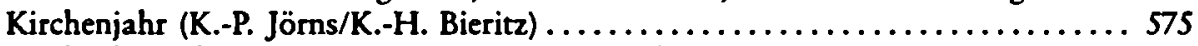

Kirchenkampf $\rightarrow$ Nationalsozialismus und Kirchen

Kirchenkunde (G. Rau).................................. 599

Kirchenleitung $\rightarrow$ Kirchenregiment, Landesherrliches, $\rightarrow$ Kirchenverwaltung/

Kirchenbehörde, $\rightarrow$ Konsistorium, $\rightarrow$ Synode

Kirchenlied (M. Jenny J. Henkys) .............................. 602

Kirchenmitgliedschaft (K.-W. Dahm) ............................ 643

Kirchenmusik (D. Schuberth) ............................... 649

Kirchenordnungen (P.F. Bradshaw/A. Sprengler-Ruppenthal/M. Plathow) .....6 662

Kirchenrecht (J. Gaudemet/M. Honecker) $\ldots \ldots \ldots \ldots \ldots \ldots \ldots \ldots \ldots \ldots \ldots \ldots \ldots \ldots$

4. Karte

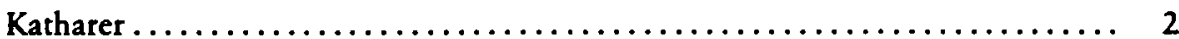

\section{Bildquellen}

Tafel 1: PKG, Suppl.bd. I, 1977, Abb. 6 u. 5; Tafel 2: ebd. Abb. 10 u. 96b; Tafel 3: ebd. Abb. 101 u. 97; Tafel 4: ebd. Abb. 100 u. 122; Tafel 5: ebd. Abb. 301b u. 8; Tafel 6: ebd. Abb. 12 u. 238; Tafel 7: PKG, Bd.V, 1969, Abb. 144 u. 268; Tafel 8: ebd., Abb. 254 u. PKG, Bd. VI, 1972, Abb. 175; Tafel 9: ebd. Abb. 336 u. PKG, Bd. VII, 1972, Abb. 375; Tafel 10: PKG V, Abb. 208 u. 166; Tafel 11: PKG VI, Abb. 35 u. PKG VII, Abb. 416; Tafel 12: Kunsthist. Inst. Berlin u. Bildarchiv Marburg; Tafel 13: Bildarchiv Marburg u. Kunsthist. Inst. Berlin; Tafel 14: Kunsthist. Inst. Berlin; Tafel 15: Bildarchiv Marburg u. Kunsthist. Inst. Berlin; Tafel 16-18 (oben): Bildarchiv Preuß. Kulturbesitz; Tafel 18 (unten): Richard Biedrzynski, Kirchen unserer Zeit, München 1958, Abb. 13; Tafel 19; ebd. Abb. 11 u. 19; Tafel 20: ebd. Abb. 41 u. VIII; Tafel 21: Albert Christ-Janer/Mary Mix Foley, Modern Church Architecture, New York u. a., o. J. Abb. S. 89 u. Ars Sacra Fennica, Helsinki 1987, S. 27; Tafel 22: KuKi 29 (3/1966) S. 112 u. 137 oben; Tafel 23: KuKi 32 (1/1969) S. 9 u. Helmut Striffler, Ein Werkbericht, hg. v. Institut f. Kirchenbau, Marburg, Mannheim 1968; Tafel 24: KuKi 3/1978, S. 123 oben u. Wallfahrtskirche Neviges, München/Zürich 1969, Umschlagbild. 